\title{
The efficacy of tourniquet assisted total knee arthroplasty on patient-reported and performance-based physical function: a randomized controlled trial protocol
}

Rasmus Lohmann-Jensen ${ }^{1,2^{*}}$, Anders Holsgaard-Larsen ${ }^{1,2}$, Claus Emmeluth ${ }^{1,2}$, Søren Overgaard ${ }^{1,2}$ and Carsten Jensen ${ }^{1,2}$

\begin{abstract}
Background: Surgical treatment of osteoarthritis with total knee arthroplasty (TKA) usually takes place in a complete bloodless field using a tourniquet. However, doing the surgery without a tourniquet may reduce muscle damage, post-surgery pain and led to improved functional rehabilitation and mobilization.

Methods/Design: A prospective, blinded, parallel-group, controlled superiority trial, with balanced randomization [1:1]. Patients aged 50 or older eligible for primary TKA for osteoarthritis will be consecutively recruited from Department of Orthopedic Surgery and Traumatology, Odense University Hospital, Denmark. A total of 80 patients will be randomly allocated to TKA with or without tourniquet application providing 40 patients for each of the two treatment arms. The tourniquet assisted TKA group will have an automatic, micro-processor-based pneumatic tourniquet inflated around the thigh during surgery. The non-tourniquet assisted TKA group will have surgery performed without application of a tourniquet. The primary aim is to compare tourniquet assisted to non-tourniquet assisted TKA on patient-reported physical function (KOOS-ADL). The secondary aim is to compare post-surgery pain, function in sports and recreation, quality of life, and performance-based physical function. The explorative outcomes include; use of pain medication, single-fiber muscle damage, and changes in mechanical muscle function. The primary endpoint will be at 3-months following surgical treatment, and the time-point for analysis of the primary outcome. However, follow-up will continue up to 1 year, and provide medium-term results. The treatment effect (difference in KOOS-ADL) will be analyzed using a random effects regression model, crude and adjusted results will be reported, if needed. Analyses will be based on the intention-to-treat (ITT). Subsequent per-protocol analysis may be necessary in the event of a substantial number of patients (> 15\%) being lost during follow-up. The number needed to treat (NNT) for a positive effect of treatment (>10 points on KOOS-ADL) will be reported.
\end{abstract}

Discussion: This is the first randomized clinical trial comparing the efficacy of tourniquet assisted TKA on patient-reported physical function supported by a range of performance-based secondary outcome measures. As such it will provide high quality evidence that may help determine whether tourniquet should be used in future TKA procedures in patients with osteoarthritis of the knee.

Trial registration: ClinicalTrials NCT01891266.

Keywords: Tourniquet, Total knee arthroplasty, Patient-reported, Performance-based, Physical function

\footnotetext{
* Correspondence: rlohmann-jensen@health.sdu.dk

'Orthopaedic Research Unit, Department of Orthopedic Surgery and

Traumatology, Odense University Hospital, Odense C, Denmark

${ }^{2}$ Institute of Clinical Research, University of Southern Denmark, Odense C,

Denmark
}

\section{Biomed Central}

(c) 2014 Lohmann-Jensen et al.; licensee BioMed Central Ltd. This is an Open Access article distributed under the terms of the Creative Commons Attribution License (http://creativecommons.org/licenses/by/2.0), which permits unrestricted use, distribution, and reproduction in any medium, provided the original work is properly credited. 


\section{Background}

Osteoarthritis (OA) is the most common joint disease and a significant cause of morbidity in middle-aged and older populations [1-3]. Knee OA is characterized by joint pain, swelling, reduced range of motion (ROM), decreased physical activity, and impaired quality of life [4]. Following the development of OA, the associated pain and disability generally increase with time $[5,6]$. In endstage knee OA, total knee arthroplasty (TKA) may be the only effective treatment available to reduce pain and restore joint mobility and function.

TKA is an invasive procedure during which damaged cartilage is removed, joint deformities are corrected and the original knee joint is replaced with an artificial joint. The recovery period typically lasts for several months and patients often experience significant pain which may be only partly relieved by analgesia. Several factors such as the post-operative inflammatory response, muscle damage, pre-existing muscle weakness and swelling may contribute to the extensive recovery period. These factors may also negatively affect physical performance in the early post-surgery period.

Tourniquets inflated above systolic blood pressure have been used by surgeons for lower limb surgery for more than a century [7-10] and are still the preferred method in elective TKA $[9,11-13]$. The rationale for tourniquet use in TKA is primarily the optimization of intraoperative visibility and reduced blood loss $[9,12,14,15]$. However, such benefits must be weighed against the potential complications which include; increased risk of direct vascular injury [13,16], nerve palsy [17-19], deep-vein thrombosis $[7,8,13,20]$ and subsequent pulmonary embolism $[7,21,22]$. Additionally, acute pulmonary edema and cardiac arrest immediately following tourniquet release have been reported $[7,22,23]$. There are also studies in the literature demonstrating that tourniquet use in TKA does not improve fixation $[24,25]$. Tourniquet use may also affect a patients' physical recovery since its use causes microscopic muscle damage, post-surgery swelling, pain, and reduced knee joint ROM [7,24-28] and a greater understanding of patient-reported outcomes following TKA using a tourniquet is required. Furthermore, tourniquet use has been reported to cause post-surgery surface electromyography changes in the quadriceps muscles [29], and has been associated with increased levels of myoglobin in plasma [30], which collectively suggests pressure induced muscle damage does occur. Finally, patients undergoing non-tourniquet assisted TKA commenced rehabilitation exercises earlier and this may improve a patient confidence in using the new joint, decrease the incidence of complications associated with immobility and improve their overall satisfaction [7,29]. The use of tourniquet for TKA is therefore controversial and well-designed randomized controlled trials are needed to provide high-level evidence to address these important issues. New trials should address aspects of recovery in physical function to fully capture the clinical implications of tourniquet use.

In response to the lack of high-level evidence the primary aim of this randomized trial is to assess the efficacy tourniquet assisted TKA on patient-reported physical function. The secondary aim is to compare post-surgery pain, function in sports and recreation, quality of life, and performance-based physical function. The explorative outcome measures include; use of pain medication, single-fiber muscle damage, and changes in mechanical muscle function. We hypothesize that non-tourniquet assisted TKA will result in superior patient-reported and physical performance-based outcome measures within the first 3-months compared with standard, tourniquet assisted, surgery.

\section{Methods/Design}

\section{Study design}

This trial will comply with CONSORT (Consolidated Standards of Reporting Trials) guidelines [31,32], and has been designed as a prospective, blinded, parallel-group, superiority trial, with balanced randomization [1:1].

\section{Participants and recruitment procedures}

Patients aged 50 years and older with an indication for TKA will be consecutively recruited from the Department of Orthopedic Surgery and Traumatology, Odense University Hospital, Denmark. The criteria for inclusion and exclusion are listed in Table 1.

On initial contact, patients will be evaluated by an orthopedic knee surgeon, who will establish the indication for surgery, and verify that all inclusion criteria and

\section{Table 1 Criteria for participants in the study}

\begin{tabular}{ll}
\hline Inclusion criteria & Exclusion criteria \\
\hline Age $>50$ & Rheumatoid arthritis \\
& BMI $>35$ \\
Able to tolerate spinal & Previous knee surgery \\
anesthesia & \\
& Malignancy \\
$\begin{array}{l}\text { Clinical and radiological knee } \\
\text { OA according to the 'Ahlbäck' }\end{array}$ & Muscle disease \\
classification system & \\
& $\begin{array}{l}\text { Deep vein thrombosis or other } \\
\text { blood coagulation disorders. }\end{array}$ \\
& Neuromuscular problems \\
& $\begin{array}{l}\text { Symptomatic bilateral OA, with } \\
\text { planned surgery of the contra } \\
\text { lateral knee within 1 year }\end{array}$ \\
& Decline or unable to participate
\end{tabular}

OA, Osteoarthritis; BMI, Body Mass Index. 
none of the exclusion criteria are met (Table 1). Next, the patients will be invited to participate in the trial, and will receive verbal and written information about the trial in accordance with the information sheet approved by the ethics committee. Patients will be given the opportunity to ask questions about the trial and if they wish to participate they will complete a standardized consent form. Patients who do not wish to make a decision regarding participating in the trial during the consultation will be given a stamped addressed envelope and will be contacted by the research nurse no later than a few 4 after the consultation. The research nurse will also ensure that participants have signed the written consent form, are randomized and scheduled for the completion of all baseline measurements. Patients declining to participate in the trial will receive standard surgery (tourniquet-assisted TKA). An independent radiologist will stage the knee osteoarthritis severity using the Ahlbäck classification system [33].

A compete overview of the recruitment procedures (enrolment, randomization, treatment allocation, followup and data analysis) is shown in Figure 1.

\section{Randomization and allocation concealment}

The randomization sequence will be computer-generated using Stata 13.0 (StataCorp, College Station, TX) statistical software with a 1:1 allocation ratio using random block sizes of 10 and 20 patients. Patients will be allocated to one of two treatments by a sequence of letters: A - referring to tourniquet assisted TKA and B - referring to nontourniquet assisted TKA using sequentially numbered opaque, sealed envelopes. The allocation sequence and preparation of the concealed envelopes will be completed by a third person (JL) not involved in the conduct of the trial.

The allocation sequence will be concealed from the group of surgeons enrolling and assessing participants. Informed consent will be obtained from the participant, in ignorance of the next assignment in the sequence, by a research nurse. To prevent subversion of the allocation sequence, name and date of birth of the participant will be written on the envelope immediately after randomization by the research nurse. Regardless of group allocation, envelopes will first be opened after enrolled patients have completed all the baseline assessments and immediately prior to the surgical intervention.

\section{Intervention}

The entire surgical procedure will be performed under spinal analgesia with a typical duration of 60-90 min and all patients, regardless of length-of-surgery, will remain in the trial population. All patients will undergo surgery using a midline and a medial parapatellar incision through the joint capsule. Patients will receive a cemented total knee arthroplasty (P.F.C. ${ }^{\circledR}$ Sigma ${ }^{\oplus}$, posterior cruciate retaining knee system, Depuy). Prior to surgery patients receive transexamic acid (Cyclocapron) to decrease bleeding. During surgery, pain management will consist of local infiltration anesthesia (300 mg naropin, $1 \mathrm{mg}$ toradol and $0.5 \mathrm{mg}$ epinephrine periarticularly). The post-surgery pain management will consist of paracetamol (1 g four times/day), contalgin (10 mg two times/day) according to our standard protocol and morphine analogs (10 mg Oxynorm) as required, with the aim of providing sufficient pain relief to facilitate early mobilization. Following discharge patients will receive one-week of antithrombosis treatment (fragmin, 5000 IE.sc). A single surgeon trained in surgery with and without the use of a tourniquet will perform both surgical procedures.

\section{Group A: the tourniquet assisted TKA group}

Patients in the tourniquet group will have surgery performed with an automatic, micro-processor-based pneumatic tourniquet (A.T.S. 2000 tourniquet system, Ref. 60-2000-101-00, Zimmer), patient-dependent size and inflated to $250 \mathrm{mmHg}$ ( $\pm 3 \mathrm{mmHg}$ pressure accuracy).

\section{Group B: the non-tourniquet assisted TKA group}

In the non-tourniquet assisted TKA group the entire operation will be performed without application of a tourniquet.

In-hospital rehabilitation will be standardized for both surgical treatments and consists of the use of crutches, range of motion and stair negotiation exercises all of which are performed under supervision of a physiotherapist. Duration of hospital stay is typically 2 to 3 days and patients are generally discharged with crutches. Following discharge the patients will be provided with a leaflet detailing exercises to continue at home as part of the ongoing rehabilitation process.

\section{Outcome measures}

A single primary outcome measure was chosen to eliminate problems with result interpretation often associated with multiplicity of analysis [34].

\section{Primary outcome measure}

The Knee injury and osteoarthritis outcome score (KOOS-ADL) KOOS is patient-reported outcome measure with good evidence of reliability, validity and responsiveness in different population with varying pathologies, injury durations and activity levels [35-37]. A 5-point Likert-scale is used and converted into a 100-point scale with zero indicating the worst possible health [35].

\section{Secondary outcome measures}

Secondary outcomes include two patient-reported and physical performance-based measures: 


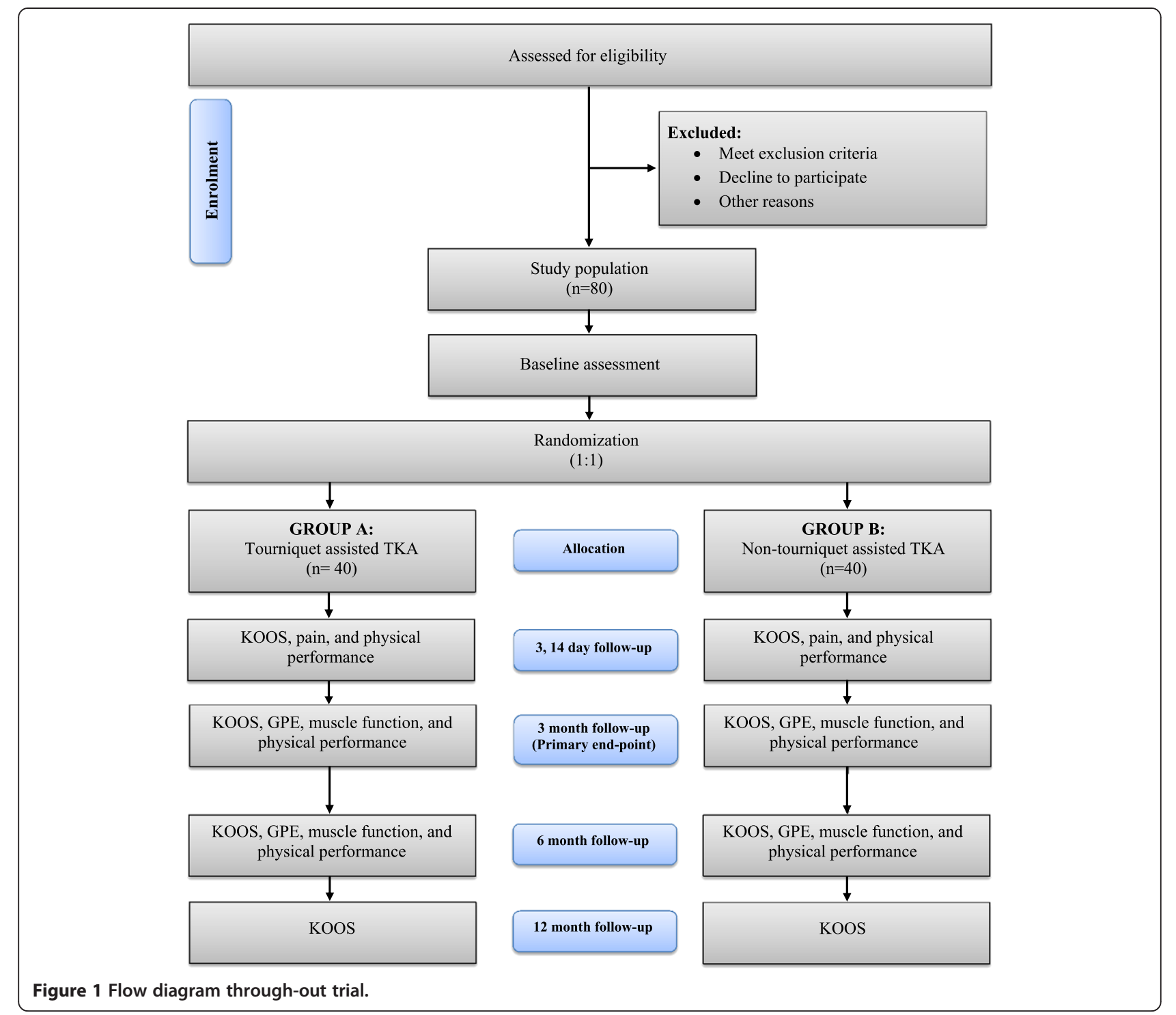

The Knee injury and osteoarthritis outcome score (KOOS) The remaining 4 subscales for pain, other symptoms, function in sport and recreation and kneerelated quality of life.

Global perceived effect (GPE) score Responsiveness is defined as the ability of a patient-reported outcome (PRO) instrument to detect changes over time in the construct to be measured [38]. For evaluating responsiveness, a GPE score, where the patients rate their condition on a 7-point Likert-scale is a recommended responder criteria [39]. The GPE questionnaire has the following seven response options: much better (3), better (2), somewhat better (1), no change (0), somewhat worse $(-1)$, worse $(-2)$ and much worse (-3). A 2-point change from 'no change' will be used as cut-off to categorize the response into: i) better, ii) no change, and iii) worse. At the 3-months and 12-months follow-up the patients will be asked to rate possible change in their condition from baseline.

Passive knee range of motion (kneeROM) The number of degrees an examiner is able to move the knee joint through its full range of motion with no active effort from the patient. Mobility is measured in degrees using a standard $(30 \mathrm{~cm})$ goniometer [40]. Inter-tester reliability $(\mathrm{r}=0.98 ; \mathrm{ICC}=0.99)$ and validity $(\mathrm{r}=0.97$; ICC $=0.98$ ) of goniometer measurements of knee joint motion have been shown [41].

The 30-s chair-stand test (30-s CST) The number of stands, from a seated position with arms folded across the chest, the patient is able to complete within a 30second time period. CST measures lower-body strength 
and is an important aspect of fitness in older adults because of its role in common everyday activities. Past studies have shown that chair stand performance correlates well with laboratory measures of lower-body strength [42], and with other indicators of interest such as walking speed, stair climbing ability and balance in older adults [43]. CST has shown good intra- and inter-rater reliability in older adults with $\mathrm{OA}(\mathrm{ICC}=0.89)[44,45]$.

$20 \mathrm{~m}$ self-paced and fast-paced walk test $(20 \mathrm{mWT})$ The time in seconds required to walk a $20 \mathrm{~m}$ distance at selfand fast-pace. Walking time have been shown to be reliable ( $\mathrm{ICC}=0.93$ and 0.98 , respectively), and valid in reflecting functional performance in knee OA patients [46]. Good agreement and excellent test-retest reliability have been demonstrated in both hip and knee OA patients [44].

Timed up-and-go test (TUG) The time in seconds needed for a patient to rise from an armchair, walk $3 \mathrm{~m}$, turn, and then return to sit on the same chair. The TUG has been shown to be reliable and valid in reflecting performance-based test of mobility $[47,48]$, and activity in daily living evaluated by a patient-reported questionnaire [49].

Self-reported pain intensity Self-reported pain intensity is measured using the continuous (or "analogue") aspect of the visual analogue scale (VAS), $(0=$ no pain and $100=$ worst possible pain). The VAS scale is reliable and valid in assessing the intensity of musculoskeletal knee pain [50].

\section{Explorative outcomes}

The explorative part of the trial investigates the changes in a range of muscle mechanical measurements; Isometric knee muscle strength (iMVC) during knee extension and flexion, leg extension power (LEP), rate of force development (RFD), force steadiness [51,52], and surface electromyography (sEMG) supported by single-leg knee bending performance and muscle biopsies.

Maximal isometric knee strength The maximal force $(\mathrm{Nm})$ generated during isometric knee extension and knee flexion. Three consecutive test contractions will be performed, and the peak value will be selected for further analysis [51]. The force-generating capacity is regarded as an essential functional outcome measure, and a prime factor to consider when examining functional limitations following TKA [53]. A comparable isometric testing device has been shown to demonstrate discriminant validity and high test-retest reliability in knee OA patients [54].

Dynamic leg extension power (LEP) The maximal power (Watt; force $\mathrm{x}$ velocity) generated by the leg extensor muscles during $1 / 2$-sec unilateral leg extension press in the Nottingham Power Rig. Each leg will be tested separately using 4-6 trial and separated by 30 -s rest. Leg extension power has been described in detail $[55,56]$ and tested for reliability in patients with knee OA [55].

The rate of force development $\left(\mathbf{R F D}_{200}\right)$ The change in force generated during the early phase of a muscle contraction (0-200 ms) [52,57]. Several activities, such as descending stairs, fast-paced walking, or prevention of fall are characterized by a limited time to generate force, and the ability to rapidly produce force is an essential functional parameter [2,58].

Force steadiness The ability to produce a given force with a minimal amount of variation, and is routinely used to quantify mechanical and neuromuscular parameters in skeletal muscle [52].

Surface Electromyography (sEMG) The surface myoelectric (sEMG) signals associated with muscle contractions will be collected on lower limb muscles; $m$. rectus femoris, $\mathrm{m}$. vastus medialis, $\mathrm{m}$. vastus lateralis, $\mathrm{m}$. biceps femoris and semitendinosus following the SENIAM guidelines [59]. EMG will be collected during iMVC and force steadiness muscle contractions. (http://www. SENIAM.org). Good test-retest reliability was reported in healthy subjects [60].

30-s single-leg knee bending (30s-KneeBend) The maximum number of bends completed within a $30 \mathrm{sec}-$ ond time period. 30s-KneeBend measures the ability to execute fast stretch shortening cycles over the knee joint. The test is valid in discriminating between the symptomatic and the non-symptomatic leg in meniscectomized patients [61] and moderate agreement and good test-retest reliability in patients with hip and knee OA has been reported [44].

Single-fiber muscle damage Muscle biopsy samples $(2 \times 100 \mathrm{mg})$ will be acquired during surgery in a subgroup of the patients $(2 \times 10)$. One sample will be collected prior to applying the tourniquet, and a second sample will be collected prior to the removal of the tourniquet. The muscle sample will be taken in every $4^{\text {th }}$ patient (e.g. patient no. 4, 8 and 12 etc.) Since the randomization sequence is concealed the unblinded surgeon will be responsible for selecting the next patient in line to ensure an equal distribution of samples drawn from each treatment group. The muscle biopsies will be performed through a small surgical incision into m. quadriceps femoris, and directly beneath the area where the tourniquet was applied. The muscle tissue samples will be frozen in liquid nitrogen, and saved for later 
analysis (single-fiber muscle isometric mechanical damage/ function; force, force/cross sectional area, $\mathrm{Ca}^{++}$-sensitivity, and fiber stiffness).

\section{Adverse events Surgery related data}

A medical record audit will be performed at 3 month postsurgery where, surgery-time, intraoperative blood loss, wound infection, deep-vein thrombosis, pulmonary embolism, time-to-ambulation, prosthetic loosening, revisions will be reviewed and summarized. Also, the degree of knee varus/valgus before and after surgery will be evaluated.

\section{Self-reported use of pain medication}

The patient's use of pain medication will be registered using a diary.

\section{Follow-up period}

Assessments will be performed pre-surgery, 3, 14 days and 3, 6, 12-months after surgery with 3-months as the primary end-point. A summary of the outcome measures and time-points can be found in Table 2.

\section{Blinding}

Blinding to treatment allocation (surgeons) is not possible due to the nature of the surgical intervention. However, the unblinded surgeon will not be involved in any care or outcome assessments. Multiple strategies will be used to maximize patients blinding in this trial. Firstly, patients will not be informed about their surgical treatment, but swelling may reveal if tourniquet was used. Second, all patients will be requested not to disclose details about their treatment to the data collectors, should they become aware of their allocation. Third, the tourniquet will first be applied when patients are under anesthesia and lightly sedated. Fourth, postsurgical care regime will be standardized for both treatment groups. Finally, identical setting, positioning of patients, masking by drape and post-surgical sham dressing will be applied.

Several strategies will be used to blind the data collector and principal investigator. The data collector responsible for baseline and follow-up assessments will be an independent person. All patient-reported outcome measures will be entered into a database using optical character recognition (OCR) scanning software and subsequently identified by trial numbers only. The principal investigator and data analyst (RLJ) will be blinded to the allocation sequence as data will be analyzed using recoded trial numbers only. The recoding of the trial identification numbers will be performed by an independent person (JL).

\section{Statistical analysis}

The primary statistical analysis will be performed on KOOS-ADL 3-month following surgery. To evaluate the treatment effect (mean difference in KOOS scores) we will employ a random or fixed effects linear regression model with point estimates [62] and both crude and adjusted results will be reported. The regression model includes the interaction between treatment and elapsed time, adjusted for pre-surgery values and assumes data is missing completely at random (MCAR) [62]. Model assumptions will be checked by residual plots. The secondary statistical analysis will include the same approach as described above for all the secondary outcomes. All analyses will be performed on the basis of intentionto-treat (ITT) [63]. However, subsequent per-protocol analysis may be necessary in the event of a substantial number of patients (> 15\%) being lost during follow-up. All data will be checked for Gaussian distribution and parametric statistics will be used were appropriate, otherwise non-parametric statistics will be applied. Finally, the number needed to treat (NNT) for a positive effect of treatment (>10 points on KOOS-ADL) will be analyzed. All statistical analyses are blinded and will be performed using Stata software (StataCorp, Texas, USA).

\section{Sample size}

Sample size estimation was performed upon the primary outcome KOOS-ADL, using one pre-surgery and 2 follow-up assessments and an estimated correlation between follow-up measurements of 0.5. Based upon data from a non-randomized, but controlled study from Sweden, a 10-point change in KOOS-ADL (SD of 18.5 pre-surgery and 10.4 post-surgery) has been selected as clinical important difference at the 3-months end-point. A sample size of $n=36$ is needed in each group $(\alpha=0.05$, $\beta=0.80)$ ). To account for possible drop-outs $n=40$ will be included in each group.

\section{Timeline and ethics}

Recruitment of patients is scheduled to begin in spring 2014 and will last for 1 year. All patients are expected to have completed the trial by spring 2016. The omission of tourniquet use is expected to only marginally increase surgery time $(\sim 5 \mathrm{~min}$.), and the increased surgery time is unlikely to increase the risk of complications. In contrast, total theatre time is expected to remain unchanged due to the time saved when application of the tourniquet is omitted. No difference, or a slight non-critical increase, in total blood loss in the non-tourniquet group is expected [15]. The trial complies with the Declaration of Helsinki and the trial has been approved by the Regional Ethics Committee of Southern Denmark (S-20110084).

\section{Discussion}

This clinical trial will evaluate the efficacy of tourniquet assisted TKA on patient-reported and performancebased physical performance As a prospective randomized 
Table 2 Summary of outcome measures to be collected in the trial with time-points

\begin{tabular}{|c|c|c|c|c|}
\hline \multirow[t]{2}{*}{ Primary outcome } & \multirow{2}{*}{$\begin{array}{c}\text { Data collection } \\
\text { instrument (unit) }\end{array}$} & \multicolumn{3}{|c|}{ Collection time-point } \\
\hline & & Baseline & Post (days) & Post (months) \\
\hline Function in daily living & KOOS & Pre & 14 & $3,6,12$ \\
\hline \multicolumn{5}{|l|}{ Secondary outcome } \\
\hline \multicolumn{5}{|l|}{ KOOS - 4 subscales } \\
\hline Pain & KOOS & Pre & 14 & $3,6,12$ \\
\hline Symptoms & KOOS & Pre & 14 & $3,6,12$ \\
\hline Sport and recreation & KOOS & Pre & 14 & $3,6,12$ \\
\hline Quality of life & KOOS & Pre & 14 & $3,6,12$ \\
\hline \multicolumn{5}{|l|}{ Questionnaire } \\
\hline Global perceived effect & GPE & & & $3,6,12$ \\
\hline \multicolumn{5}{|l|}{ Bilateral physical Performance } \\
\hline Knee range of motion (KneeROM) & Goniometer $\left(^{\circ}\right)$ & Pre & 3,14 & 3,6 \\
\hline 30-s chair-stand test (30s CRT) & (Number) & Pre & 3,14 & 3,6 \\
\hline 20-m self- and fast-paced walk test ( $20 \mathrm{mWT})$ & Stopwatch (s) & Pre & 3,14 & 3,6 \\
\hline Timed up-and-go test (TUG) & Stopwatch (s) & Pre & 3,14 & 3,6 \\
\hline \multicolumn{5}{|l|}{ Explorative outcomes } \\
\hline \multicolumn{5}{|l|}{ Mechanical muscle function } \\
\hline Maximal isometric knee strength (iMVC) & Strain-gauge (Nm) & Pre & & 3,6 \\
\hline Dynamic leg extension power & $(\mathrm{W})$ & Pre & & 3,6 \\
\hline Rate of force development (RFD) & $(\mathrm{Nm} / \mathrm{sec})$ & Pre & & 3,6 \\
\hline Surface electromyography (EMG) & Myon (mV) & Pre & & 3,6 \\
\hline Force steadiness & $(\mathrm{Nm})$ & Pre & & 3,6 \\
\hline \multicolumn{5}{|l|}{ Unilateral physical performance } \\
\hline 30-s single-leg knee bending (30s-KneeBend) & (Number) & Pre & & 3,6 \\
\hline \multicolumn{5}{|l|}{ Pain } \\
\hline Self-reported use of pain medication & (Quantity) & Pre & $1-14$ & \\
\hline Self-reported pain (VAS) & VAS & Pre & $1-14$ & \\
\hline \multicolumn{5}{|l|}{ Biopsies } \\
\hline Muscle biopsy & & \multicolumn{3}{|c|}{ Prior to applying and removal of the tourniquet } \\
\hline
\end{tabular}

controlled trial the results of this study will provide a high level of evidence on the clinical implications of using a tourniquet during TKA. The primary end point, at 3-month, is a sufficiently long timeframe for the relevant clinical improvements between groups to show and yet short enough to assume that patients can recall their baseline condition. As part of the design, a single senior surgeon will perform the surgical procedures in both groups. The experience of the surgeon in both tourniquet-assisted and non-tourniquet TKA and use of random block size randomization will eliminate bias due to learning. Additionally use of a single surgeon will ensure we are comparing the TKA procedure rather than the skill of different surgeons.

The key aim of TKA in patients with OA is to relieve pain and improve function, both of which are patient reported outcome (PROs) measures reflected in the design of this trial and recommended outcome measures by the Osteoarthritis Research Society International (OARSI) and Outcome Measures in Rheumatology and Clinical Trials (OMERACT) group [39]. Ideally, changes in physical function following TKA should be evaluated using a combination of patient-reported outcomes (PROs) and performance-based outcome measures [39,45] as they are complementary rather than competing [64,65] and capture different constructs of physical function [66,67]. In addition, responsiveness of a PROs ability to detect when patients are undergoing relevant clinical changes can be assessed using a global response evaluation (GPE), and a GPE questionnaire is a recommended part of the evaluation of a treatment in clinical trials [38,39]. To accommodate these recommendations, the patients in the 
present trial will be asked to rate their current knee condition pre-surgery (baseline), and at each follow-up (KOOS) and also rate changes in their condition since baseline (GPE). These two patient-reported outcome measures (KOOS and GPE) will be supported by assessor-observed performance-based tests of physical function, hence evaluating, what 'patients can do' rather than what they 'perceive they can do' [67].

According to WHOs classification of Functioning, Disability and Health (ICF) physical function can be classified as the ability to "perform daily activities" [68]. Hence, to fully capture physical function in patients following joint replacement, performance-based testing should represent a variety of physical function domains (activity themes) representing the following five daily activities; walking, sit-to-stand, ambulatory transitions, stair negotiation, and endurance [45]. The current trial uses the $2 \times 20 \mathrm{~m}$ fast-paced walk test, the 30 -s chairstand test, and the timed up-and-go test evaluate; walking, sit-to-stand, and ambulatory transition activity domains, repetitively. Despite the importance of also evaluating stair negotiation activity, no specific test is planned in the trial for two reasons. First, no recommendations about specific performance-based tests of physical function, with good measurement-property evidence (reliability, validity, responsiveness, and interpretability), is currently available. Second, the feasibility of stair negotiation tests is largely dependent on the environmental setting making them unsuitable for clinical trials at present [45]. Likewise, no test for evaluating endurance activity has been planned for this trial due to the practical feasibilities issues associated with the six-minute walk test, e.g. patients are unable to walk for six minutes and exhausted following surgery. Further, endurance activity evaluation may be compromised in the early postsurgery phase since pain and swelling, rather than endurance, could limit patients' performance. Consequently the current trial evaluates three primary activity themes using a core set of performance-based tests recommended by OARSI [45], and from this core set of tests efficient comparisons of treatment outcomes across published literature can be made.

Evaluating the domain of pain or physical function in patients following joint replacement surgery is important [39]. However, the decision to nominate physical function as primary outcome was due to two reasons. The KOOS questionnaire evaluates physical function in a variety of daily activities, and has an extensive subscale (KOOS-ADL, 17 items) devoted to evaluate physical function compared to the pain-subscale (KOOS-PS, 10 items). Additionally, WOMAC and KOOS are the most common PROs used to capture patient relevant information, relating to the impact of interventions in clinical research [69]. WOMAC scores can subsequently be calculated based on data collected using the KOOS questionnaire, thus making efficient comparisons across published literature possible.

Essential explorative outcome measures are also included in the present trial to study muscle mechanics and potential microscopic muscle damage. Studying muscle mechanics is clinical relevant in understanding body structure $[51,70]$ and the recovery of muscle strength and power are likely to be compromised in tourniquet assisted TKA patients. The theoretical disadvantages of tourniquet application on muscle mechanics is supported by one randomized trial [7] and two non-controlled studies in which reduced muscle function and neurological impairment have been reported [29,71]. However, such findings should be confirmed in high-quality trials using randomized designs.

A number of other factors may also influence the effects of tourniquets [15]. It's been suggested that the tourniquet position may be of clinical importance [72] but also cuff width, cuff pressure, time of cuff deflation (cementation of the prosthesis, would closure or application of dressing) must be considered factors that may affect outcome, and should be recorded [15]. Other factors include use of thromboprophylaxis, girth/circumference of thigh, and patient blood pressure. Data on these variables will be collected to insure uniform presentation of data in peer-reviewed papers $[9,15]$.

\section{Conclusion}

We have designed a randomized clinical trial with the main purpose of investigating the efficacy of tourniquet assisted TKA on patient-reported physical function, an outcome which will be supported by a range of performance-based secondary outcome measures. This part of the trial will provide results with high-level evidence and may help to determine whether tourniquet should be used in future TKA procedures for those with severe osteoarthritis of the knee. The explorative part of the trial could also provide further understanding of the underlying neurological and muscle mechanical impairments associated with use of tourniquet during TKA surgery. The results will be submitted to a peer-reviewed international journal for publication irrespective of the outcome in accordance with the CONSORT guidelines for reporting of clinical trials.

\footnotetext{
Abbreviations

ADL: Activities of daily living; GPE: Global perceived effect; KOOS: The knee disability and osteoarthritis outcome score; iMVC: Isometric maximal voluntary contraction; QoL: Quality of life; TKA: Total knee arthroplasty; VAS: Visual analog scale; LEP: Leg extension power.
}

Competing interests

The authors declare that they have no competing interests. 


\section{Authors' contributions}

$\mathrm{RLJ}$ will manage the coordination of the trial. RLJ and $\mathrm{CJ}$ were responsible for drafting this paper. $\mathrm{AHL}, \mathrm{SO}$ and $\mathrm{CJ}$ conceived the project while SO/CJ/RLJ procured the project funding. CE will be responsible for planning and execution of the knee arthroplasty. All authors provided intellectual input on the design, feedback on drafts of this protocol, and approved the final manuscript.

\section{Acknowledgements}

This trial is funded by The Region of Southern Denmark and Zealand, Bevica Foundation, and The Danish Rheumatism Association. None of the sponsors have any role in the trial other than to provide funding. We would like to thank Jens Lauritsen, who designed and provided the randomization schedule.

Received: 5 July 2013 Accepted: 20 March 2014

Published: 29 March 2014

\section{References}

1. Brooks PM: Impact of osteoarthritis on individuals and society: how much disability? Social consequences and health economic implications. Curr Opin Rheumatol 2002, 14:573-577.

2. Izquierdo M, Aguado X, Gonzalez R, Lopez JL, Hakkinen K: Maximal and explosive force production capacity and balance performance in men of different ages. Eur J Appl Physiol Occup Physiol 1999, 79:260-267.

3. Salaffi F, Carotti M, Stancati A, Grassi W: Health-related quality of life in older adults with symptomatic hip and knee osteoarthritis: a comparison with matched healthy controls. Aging Clin Exp Res 2005, 17:255-263.

4. Buckwalter JA, Martin JA: Osteoarthritis. Adv Drug Deliv Rev 2006, 58:150-167

5. Lapsley HM, March LM, Tribe KL, Cross MJ, Brooks PM: Living with osteoarthritis: patient expenditures, health status, and social impact. Arthritis Rheum 2001, 45:301-306.

6. Leigh JP, Seavey W, Leistikow B: Estimating the costs of job related arthritis. J Rheumatol 2001, 28:1647-1654

7. Abdel-Salam A, Eyres KS: Effects of tourniquet during total knee arthroplasty. A prospective randomised study. J Bone Joint Surg (Br) 1995, 77:250-253.

8. Fukuda A, Hasegawa M, Kato K, Shi D, Sudo A, Uchida A: Effect of tourniquet application on deep vein thrombosis after total knee arthroplasty. Arch Orthop Trauma Surg 2007, 127:671-675.

9. Smith TO, Hing CB: A meta-analysis of tourniquet assisted arthroscopic knee surgery. Knee 2009, 16:317-321.

10. Tetro AM, Rudan JF: The effects of a pneumatic tourniquet on blood loss in total knee arthroplasty. Can J Surg 2001, 44:33-38.

11. Aglietti $P$, Baldini A, Vena LM, Abbate R, Fedi S, Falciani M: Effect of tourniquet use on activation of coagulation in total knee replacement. Clin Orthop Relat Res 2000, Feb:169-177.

12. Johnson DS, Stewart $H$, Hirst $P$, Harper $N J$ : Is tourniquet use necessary for knee arthroscopy? Arthroscopy 2000, 16:648-651.

13. Wakankar HM, Nicholl JE, Koka R, D'Arcy JC: The tourniquet in total knee arthroplasty. A prospective, randomised study. J Bone Joint Surg (Br) 1999, 81:30-33.

14. Daniel DM, Lumkong G, Stone ML, Pedowitz RA: Effects of tourniquet use in anterior cruciate ligament reconstruction. Arthroscopy 1995, 11:307-311

15. Smith $T O$, Hing CB: Is a tourniquet beneficial in total knee replacement surgery? A meta-analysis and systematic review. Knee 2010, 17:141-147.

16. Irving GA, Noakes TD: The protective role of local hypothermia in tourniquet-induced ischaemia of muscle. J Bone Joint Surg (Br) 1985, 67:297-301.

17. Newman RJ: Metabolic effects of tourniquet ischaemia studied by nuclear magnetic resonance spectroscopy. J Bone Joint Surg (Br) 1984, 66:434-440.

18. Olivecrona C, Blomfeldt R, Ponzer S, Stanford BR, Nilsson BY: Tourniquet cuff pressure and nerve injury in knee arthroplasty in a bloodless field: a neurophysiological study. Acta Orthop 2013, 84:159-164.

19. Sapega AA, Heppenstall RB, Chance B, Park YS, Sokolow D: Optimizing tourniquet application and release times in extremity surgery. A biochemical and ultrastructural study. J Bone Joint Surg Am 1985, 67:303-314.
20. Tai TW, Lin CJ, Jou IM, Chang CW, Lai KA, Yang CY: Tourniquet use in total knee arthroplasty: a meta-analysis. Knee Surg Sports Traumatol ArthrosC 2011, 19:1121-1130.

21. Nishiguchi M, Takamura N, Abe Y, Kono M, Shindo H, Aoyagi K: Pilot study on the use of tourniquet: a risk factor for pulmonary thromboembolism after total knee arthroplasty? Thromb Res 2005, 115:271-276.

22. McGrath BJ, Hsia J, Epstein B: Massive pulmonary embolism following tourniquet deflation. Anesthesiology 1991, 74:618-620.

23. Gielen M: Cardiac arrest after tourniquet release. Can J Anaesth 1991 38:541.

24. Molt M, Harsten A, Toksvig-Larsen S: The effect of tourniquet use on fixation quality in cemented total knee arthroplasty a prospective randomized clinical controlled RSA trial. Knee 2013, Oct 24 doi: 10.1016/j.knee.2013.10.008. [Epub ahead of print].

25. Ledin H, Aspenberg P, Good L: Tourniquet use in total knee replacement does not improve fixation, but appears to reduce final range of motion Acta Orthop 2012, 83:499-503.

26. Klenerman $L$ : Is a tourniquet really necessary for knee replacement? J Bone Joint Surg (Br) 1995, 77:174-175.

27. Silver R, de la Garza J, Rang M, Koreska J: Limb swelling after release of a tourniquet. Clin Orthop Relat Res 1986, Maj:86-89.

28. Tibrewal SB: The pneumatic tourniquet in arthroscopic surgery of the knee. Int Orthop 2001, 24:347-349.

29. Saunders KC, Louis DL, Weingarden SI, Waylonis GW: Effect of tourniquet time on postoperative quadriceps function. Clin Orthop Relat Res 1979, Sep:194-199.

30. Jorgensen HR: Myoglobin release after tourniquet ischemia. Acta Orthop Scand 1987, 58:554-556.

31. Moher D, Hopewell S, Schulz KF, Montori V, Gotzsche PC, Devereaux PJ, Elbourne D, Egger M, Altman DG: CONSORT, explanation and elaboration: updated guidelines for reporting parallel group randomised trials. J Clin Epidemiol 2010, 2010(63):e1-e37.

32. Schulz KF, Altman DG, Moher D: CONSORT, statement: updated guidelines for reporting parallel group randomised trials. J Clin Epidemiol 2010, 2010(63):834-840.

33. Ahlback S: Osteoarthrosis of the knee. A radiographic investigation. Acta Radiol Diagn 1968, suppl. 277(7):72.

34. Schulz KF, Grimes DA: Multiplicity in randomised trials I: endpoints and treatments. Lancet 2005, 365:1591-1595.

35. Knee Disability and Osteoarthritis Outcome Score (KOOS). [http://www.koos.nu]

36. Roos EM, Lohmander LS: The Knee injury and Osteoarthritis Outcome Score (KOOS): from joint injury to osteoarthritis. Health Qual Life Outcomes 2003, 1:64.

37. Roos EM, Roos HP, Ekdahl C, Lohmander LS: Knee injury and Osteoarthritis Outcome Score (KOOS)-validation of a Swedish version. Scand J Med SCi Sports 1998, 8:439-448.

38. Mokkink LB, Terwee CB, Patrick DL, Alonso J, Stratford PW, Knol DL, Bouter LM, de Vet HC: The COSMIN checklist for assessing the methodological quality of studies on measurement properties of health status measurement instruments: an international Delphi study. Qual Life Res 2010, 19:539-549.

39. Bellamy N, Kirwan J, Boers M, Brooks P, Strand V, Tugwell P, Altman R, Brandt K, Dougados M, Lequesne M: Recommendations for a core set of outcome measures for future phase III clinical trials in knee, hip, and hand osteoarthritis. Consensus development at OMERACT III. J Rheumatol 1997, 24:799-802.

40. Poulsen E, Christensen HW, Penny JO, Overgaard S, Vach W, Hartvigsen J: Reproducibility of range of motion and muscle strength measurements in patients with hip osteoarthritis - an inter-rater study. BMC Musculoskelet Disord 2012, 13:242

41. Gogia PP, Braatz JH, Rose SJ, Norton BJ: Reliability and validity of goniometric measurements at the knee. Phys Ther 1987, 67:192-195

42. Jones CJ, Rikli RE, Beam WC: A 30-s chair-stand test as a measure of lower body strength in community-residing older adults. Res Q Exerc Sport 1999, 70:113-119.

43. Bohannon RW: Sit-to-stand test for measuring performance of lower extremity muscles. Percept Mot Skills 1995, 80:163-166.

44. Villadsen A, Roos EM, Overgaard S, Holsgaard-Larsen A: Agreement and reliability of functional performance and muscle power in 
patients with advanced osteoarthritis of the hip or knee. Am J Phys Med Rehabil 2012, 91:401-410.

45. Dobson F, Hinman RS, Roos EM, Abbott JH, Stratford P, Davis AM, Buchbinder R, Snyder-Mackler L, Henrotin Y, Thumboo J, Hansen P, Bennell KL: OARSI recommended performance-based tests to assess physical function in people diagnosed with hip or knee osteoarthritis. Osteoarthritis Cartilage 2013, 21:1042-1052.

46. Marks R: Reliability and validity of self-paced walking time measures for knee osteoarthritis. Arthritis Care Res 1994, 7:50-53.

47. Podsiadlo D, Richardson S: The timed "Up \& Go": a test of basic functional mobility for frail elderly persons. J Am Geriatr Soc 1991, 39:142-148.

48. Yeung TS, Wessel J, Stratford PW, MacDermid JC: The timed up and go test for use on an inpatient orthopaedic rehabilitation ward. J Orthop Sports Phys Ther 2008, 38:410-417.

49. Rikli RE, Jones $\mathrm{CJ}$ : Development and validation of criterion-referenced clinically relevant fitness standards for maintaining physical independence in later years. Gerontologist 2013, 53:255-267.

50. Boeckstyns ME, Backer M: Reliability and validity of the evaluation of pain in patients with total knee replacement. Pain 1989, 38:29-33.

51. Jensen $C$, Aagaard $P$, Overgaard $S$ : Recovery in mechanical muscle strength following resurfacing vs standard total hip arthroplasty - a randomised clinical trial. Osteoarthritis Cartilage 2011, 19:1108-1116.

52. Aagaard P, Simonsen EB, Andersen $J$, Magnusson P, Dyhre-Poulsen P: Increased rate of force development and neural drive of human skeletal muscle following resistance training. J Appl Physiol 2002, 93:1318-1326.

53. Mizner RL, Snyder-Mackler L: Altered loading during walking and sit-to-stand is affected by quadriceps weakness after total knee arthroplasty. J Orthop Res 2005, 23:1083-1090.

54. Fransen M, Crosbie J, Edmonds J: Isometric muscle force measurement for clinicians treating patients with osteoarthritis of the knee. Arthritis Rheum 2003, 49:29-35.

55. Robertson S, Frost $\mathrm{H}$, Doll H, O'Connor Jj: Leg extensor power and quadriceps strength: an assessment of repeatability in patients with osteoarthritic knees. Clin Rehabil 1998, 12:120-126.

56. Bassey EJ, Short AH: A new method for measuring power output in a single leg extension: feasibility, reliability and validity. Eur J Appl Physiol Occup Physiol 1990, 60:385-390

57. Maffiuletti NA, Bizzini M, Widler K, Munzinger U: Asymmetry in quadriceps rate of force development as a functional outcome measure in TKA. Clin Orthop Relat Res 2010, 468:191-198.

58. Pijnappels M, Bobbert MF, van Dieen JH: Control of support limb muscles in recovery after tripping in young and older subjects. Exp Brain Res 2005, 160:326-333.

59. Surface ElectroMyoGraphy for the Non-Invasive Assessment of Muscles (SENIAM). [http://www.seniam.org]

60. Burden A: How should we normalize electromyograms obtained from healthy participants? What we have learned from over 25 years of research. J Electromyogr Kinesiol 2010, 20:1023-1035.

61. Bremander $A B, D a h l L L$, Roos EM: Validity and reliability of functional performance tests in meniscectomized patients with or without knee osteoarthritis. Scand J Med Sci Sports 2007, 17:120-127.

62. Rabe-Hesketh S, Skrondal A: Multilevel and Longitudinal Modeling Using Stata. College Station, Texas: Stata Press; 2008.

63. Sainani KL: Making sense of intention-to-treat. PM R 2010, 2:209-213.

64. Jordan KP, Wilkie R, Muller S, Myers H, Nicholls E: Measurement of change in function and disability in osteoarthritis: current approaches and future challenges. Curr Opin Rheumatol 2009, 21:525-530.

65. Wright AA, Hegedus EJ, Baxter GD, Abbott JH: Measurement of function in hip osteoarthritis: developing a standardized approach for physical performance measures. Physiother Theory Pract 2011, 27:253-262.

66. Stratford PW, Kennedy DM: Performance measures were necessary to obtain a complete picture of osteoarthritic patients. J Clin Epidemiol 2006, 59:160-167.

67. Terwee CB, Mokkink LB, Steultjens MP, Dekker J: Performance-based methods for measuring the physical function of patients with osteoarthritis of the hip or knee: a systematic review of measurement properties. Rheumatology (Oxford) 2006, 45:890-902.

68. International Classification of Functioning, Disability and Health (ICF). [http://www.who.int/classifications/icf/en]
69. Bellamy N, Buchanan WW, Goldsmith CH, Campbell J, Stitt LW: Validation study of WOMAC: a health status instrument for measuring clinically important patient relevant outcomes to antirheumatic drug therapy in patients with osteoarthritis of the hip or knee. J Rheumatol 1988, 15:1833-1840.

70. Rasch A, Bystrom AH, Dalen N, Berg HE: Reduced muscle radiological density, cross-sectional area, and strength of major hip and knee muscles in 22 patients with hip osteoarthritis. Acta Orthop 2007, 78:505-510.

71. Krebs DE: Isokinetic, electrophysiologic, and clinical function relationships following tourniquet-aided knee arthrotomy. Phys Ther 1989, 69:803-815.

72. Finsen $\mathrm{V}$, Kasseth AM: Tourniquets in forefoot surgery: less pain when placed at the ankle. J Bone Joint Surg (Br) 1997, 79:99-101.

doi:10.1186/1471-2474-15-110

Cite this article as: Lohmann-Jensen et al:: The efficacy of tourniquet assisted total knee arthroplasty on patient-reported and performancebased physical function: a randomized controlled trial protocol. BMC Musculoskeletal Disorders 2014 15:110.

\section{Submit your next manuscript to BioMed Central and take full advantage of:}

- Convenient online submission

- Thorough peer review

- No space constraints or color figure charges

- Immediate publication on acceptance

- Inclusion in PubMed, CAS, Scopus and Google Scholar

- Research which is freely available for redistribution 